BMJ Open

Diabetes

Research

\& Care

\title{
Association of physical activity and sedentary behavior with type 2 diabetes and glycemic traits: a two-sample Mendelian randomization study
}

\author{
Christa Meisinger (10 ,1,2 Jakob Linseisen (1) , ,2 Michael Leitzmann, ${ }^{3}$ \\ Hansjoerg Baurecht, ${ }^{3}$ Sebastian Edgar Baumeister ${ }^{1,2}$
}

To cite: Meisinger C, Linseisen J, Leitzmann M, et al. Association of physical activity and sedentary behavior with type 2 diabetes and glycemic traits: a two-sample Mendelian randomization study. BMJ Open Diab Res Care 2020;8:e001896. doi:10.1136/ bmjdrc-2020-001896

- Supplemental material is published online only. To view please visit the journal online (http://dx.doi.org/10.1136/ bmjdrc-2020-001896).

HB and SEB contributed equally.

Received 10 September 2020 Revised 19 0ctober 2020 Accepted 11 November 2020

Check for updates

(c) Author(s) (or their employer(s)) 2020. Re-use permitted under CC BY-NC. No commercial re-use. See rights and permissions. Published by BMJ.

For numbered affiliations see end of article.

Correspondence to Dr Christa Meisinger; christa.meisinger@helmholtzmuenchen.de

\section{ABSTRACT}

Introduction Observational studies suggest that physical activity lowers and sedentary behavior increases the risk of type 2 diabetes. Despite of some supportive trial data for physical activity, it is largely unresolved whether these relations are causal or due to bias.

Objective We investigated the associations between accelerometer-based physical activity and sedentary behavior with type 2 diabetes and several glycemic traits using two-sample Mendelian randomization analysis. Research design and methods Single nucleotide polymorphisms (SNPs) associated at $p<5 \times 10^{-8}$ with accelerometer-based physical activity average accelerations, vigorous physical activity (fraction of accelerations $>425$ milligravities), and sedentary behavior (metabolic equivalent task $\leq 1.5$ ) in a genomewide analysis of the UK Biobank served as instrumental variables.

Outcomes Type 2 diabetes, hemoglobin A1c (HbA1C), fasting glucose, homeostasis model assessment of beta-cell function (HOMA-B), and homeostasis model assessment of insulin resistance (HOMA-IR).

Results Physical activity and sedentary behavior were unrelated to type 2 diabetes, $\mathrm{HbA1c}$, fasting glucose, HOMA-B, and HOMA-IR. The inverse variance weighted ORs per SD increment for the association between average accelerations and vigorous physical activity with type 2 diabetes were $1.00(95 \% \mathrm{Cl} 0.94$ to $1.07, \mathrm{p}=0.948)$ and 0.83 (95\% Cl 0.56 to $1.23, p=0.357$ ), respectively. These results were confirmed by sensitivity analyses using alternative MR-methods to test the robustness of our findings.

Conclusions Based on these results, genetically predicted objectively measured average or vigorous physical activity and sedentary behavior is not associated with type 2 diabetes risk or with glycemic traits in the general population. Further research is required to deepen the understanding of the biological pathways of physical activity.

\section{INTRODUCTION}

Type 2 diabetes is one of the most frequent non-communicable diseases, with an estimated 463 million cases worldwide in 2019 ; this number is projected to increase to 578

\section{Significance of this study}

What is already known about this subject?

- Based on randomized controlled trials mostly conducted in people with high risk for diabetes, there is no clear evidence that physical activity alone or diet alone compared with standard treatment influences the risk for type 2 diabetes.

What are the new findings?

- In a two-sample Mendelian randomization study, neither genetically predicted objectively measured average and vigorous physical activity nor sedentary behavior were significantly associated with type 2 diabetes and glycemic traits in the general population.

How might these results change the focus of research or clinical practice?

- Further investigation is necessary to deepen the understanding of the biologic pathways of physical activity.

million by $2030 .^{1}$ A variety of observational cohort studies found an inverse association between physical activity (PA) and type 2 diabetes, while only a few investigations reported no association. ${ }^{2}$ Studies showed a reduced risk with greater moderate and vigorous activity, but data were less consistent for low intensity activity such as walking. ${ }^{2}$ Subsequent studies focused on specific types of PA and possible dose-response relationships. ${ }^{2}$ In addition, meta-analyses of intervention studies revealed that exercise programs induce changes in glycemic traits such as fasting blood glucose and glycated hemoglobin levels. ${ }^{3}$ However, the beneficial effect of PA seems to depend on the duration of exercise and not on the type and intensity of activity. $^{3}$

Previous observational studies have relied on self-report measures of $\mathrm{PA}$, which are 
prone to recall and response biases and may attenuate 'true' associations with the outcome. ${ }^{45}$ There is evidence that self-reported and objective measures of PA can yield discrepant estimates. ${ }^{4-8}$ Large epidemiologic studies usually lack objective measurements of PA; however, the UK Biobank is an exception. In approximately 100000 study participants, PA was measured using a wrist accelerometer that study participants wore for 7 days. ${ }^{9}$ Although objective methods to measure PA help address measurement error, observational studies can be subject to other biases including residual confounding and reverse causality.

Mendelian randomization (MR) may provide another line of evidence concerning the roles of PA and sedentary behaviors (SBs) in type 2 diabetes. MR tests the effects of PA on type 2 diabetes using genetic variants as instruments that are explicitly associated with the exposure PA and exert an effect on type 2 diabetes only via the exposure. Because variants are randomly allocated from parents to offspring at conception, they are less susceptible to environmental confounding and reverse causation than traditional observational studies. ${ }^{10}$ Because genetic variants instrument for long-term levels of PA, regression dilution bias is less likely in MR studies. ${ }^{11}$ Moreover, objectively measured PA is more heritable ${ }^{12}$ than self-reported PA and thus more powerfully instrumented by single nucleotide polymorphism (SNPs) in the MR context. ${ }^{13}$ We performed two-sample MR analyses to investigate the relationship between accelerometer-based average accelerations, vigorous PA (fraction of accelerations $>425$ milligravities), and SB with type 2 diabetes and glycemic traits, namely hemoglobin A1c (HbAlc), fasting blood glucose, homeostasis model assessment of beta-cell function (HOMA-B), and homeostasis model assessment of insulin resistance (HOMA-IR).

\section{METHODS}

The study design had five components: (1) identification of genetic variants to serve as instrumental variables for PA and SB; (2) acquisition of instrumenting SNP outcome summary data from genome-wide association studies of type 2 diabetes, HbAlc levels, fasting glucose levels, HOMA-B, and HOMA-IR; (3) harmonization of SNP exposure and SNP outcome datasets; (4) statistical analysis; and (5) evaluation of MR analysis assumptions and sensitivity analyses.

\section{Assessment of PA in UK Biobank}

Data regarding different types of PA were gathered in the UK Biobank, a large prospective cohort study including approximately 500000 men and women (ages 40-69 years) living in the UK. Recruitment from 22 centers across the UK was performed between 2006 and 2010. ${ }^{14}$ All study participants provided written informed consent.

In approximately $100 \quad 000$ study participants, accelerometer-based PA data (Axivity AX3 wrist-worn accelerometer) were gathered. ${ }^{9}$ We used genetic variants proxying two accelerometer-based PA measures: average accelerations (mean acceleration in milligravities) and the fraction of accelerations $>425$ milligravities, ${ }^{12}$ the latter corresponding to an equivalent of vigorous PA ( $\geq 6$ metabolic equivalent tasks (METs)). Accelerometerbased SB was defined as a MET $\leq 1.50 .^{15}$

\section{Selection of instrumental variables for PA}

Most UK Biobank participants were genotyped with the Affymetrix UK Biobank Axiom Array (Santa Clara, California, USA), while about $10 \%$ were genotyped with the Affymetrix UK BiLEVE Axiom Array. ${ }^{16}$

We initially selected eight SNPs associated with average accelerations and eight SNPs associated with vigorous $\mathrm{PA}$ at a genome-wide significance level $\left(\mathrm{p}<5 \times 10^{-8}\right)$ in 91 084 UK Biobank participants. ${ }^{12}$ In addition, we selected six SNPs associated with SB at $\mathrm{p}<5 \times 10^{-8} .{ }^{15}$ We looked up each instrument SNP and its proxies $\left(r^{2}>0.8\right)$ in the PhenoScanncer genome-wide association study (GWAS) database (http://phenoscanner.medschl.cam.ac.uk $)^{17}$ to assess any previous associations $\left(\mathrm{p}<1 \times 10^{-8}\right)$ with the outcomes or potential confounders. Smoking was considered a relevant confounder. ${ }^{18}{ }^{19}$ We identified one of the SNPs for average accelerations (rs28749810) nominally associated with type 2 diabetes and the metabolic syndrome, respectively. After removing this SNP, seven, eight, and six SNPs were used as instrumental variables for average accelerations, vigorous $\mathrm{PA}$, and $\mathrm{SB}$ in the primary analysis.

\section{GWAS summary data for outcomes}

Summary data for the associations of genetic variants with type 2 diabetes were obtained from a GWAS metaanalysis of 32 studies including 898130 individuals of European ancestry from the Diabetes Genetics Replication And Meta-analysis (DIAGRAM Consortium; 74 124 type 2 diabetes cases and 824006 controls).$^{20}$ For HbA1c, summary data from a publication by Wheeler et $a l^{21}$ based on 123665 diabetes-free participants' data from the Meta-Analyses of Glucose and Insulin-related Traits Consortium (MAGIC) were used. Summary data for fasting glucose (sample size $\mathrm{n}=58$ 074), HOMA-B $(\mathrm{n}=36466)$, and HOMA-IR ( $\mathrm{n}=37$ 037) were drawn from publications by Manning $e t a l^{22}$ and Dupuis $e t a l^{23}$ based on data of diabetes-free individuals of European ancestry from the MAGIC. The GWAS for the outcomes did not include data from the UK Biobank. Online supplemental tables 2-4 provide associations of genome-wide significant harmonized SNPs for accelerometer-based PA and SB with type 2 diabetes and glycemic traits.

\section{Data availability}

The present study is based on summary-level data that have been made publicly available. In all original studies, ethical approval had been obtained. The summary statistics for the PA and SB GWAS is available at https:/ / klimentidis.lab.arizona.edu/content/data and at https://doi. org/10.5287/bodleian:yJp6zZmdj. The summary data 
for the type 2 diabetes GWAS are available at http:// diagram-consortium.org/downloads.html, while for the HbA1c, fasting glucose, HOMA-B and HOMA-IR GWASs, summary data are available at www.magicinvestigators. org/downloads.

\section{Statistical power}

The a priori statistical power for the binary trait was calculated according to Burgess ${ }^{24}$ and for continuous traits according to Deng. ${ }^{25}$

The eight SNPs for average accelerations explained $0.25 \%$, and the eight SNPs for vigorous activity explained $0.25 \%$ of the phenotypic variance. The analyses were sufficiently powered to identify associations between the different exposure variables and outcomes (online supplemental table 6).

\section{Statistical analyses}

The principal analysis was conducted using a multiplicative random effects inverse-variance weighted (IVW) method, which allows for each SNP to have different mean effects. ${ }^{26}$ The results for the outcome type 2 diabetes are presented as ORs and 95\% CIs per 1 SD increment in average accelerations and vigorous $\mathrm{PA}$ or $\mathrm{SB}$. One SD of objectively measured PA in the UK Biobank study has been reported to be approximately 8 milligravities (or $0.08 \mathrm{~m} / \mathrm{s}^{2}$ ) of acceleration in a mean $5 \mathrm{~s}$ window of analyzed accelerometer data. ${ }^{15}$ A 1-SD increment in average accelerations ( 8.14 milligravities or $\left.0.08 \mathrm{~m} / \mathrm{s}^{2}\right)$ approximates to about 3 MET-hour/day, ${ }^{27}$ with one MET equal to the metabolic cost of sitting quietly. ${ }^{28}$ The results for the continuous outcomes are presented as $\beta$-estimates and $95 \%$ CIs per SD of objectively measured PA or SB.

One key assumption for IVW to produce a valid estimate is that there is no other way SNPs could affect the outcome than through the exposure. Violations of this assumption through horizontal pleiotropy can introduce bias, whereby the instruments exert an effect on the outcome independent of the exposure. To examine possible violations of this assumption, we checked each candidate SNP and its proxies $\left(\mathrm{r}^{2}>0.8\right)$ in PhenoScanner (online supplemental table 5) for previously reported associations $\left(\mathrm{p}<5 \times 10^{-8}\right)$ with confounders. The presence of pleiotropy was further investigated using betweeninstrument heterogeneity of the IVW estimates based on a modified Cochran's $Q$ statistic (online supplemental table 7). If the pleiotropy is 'balanced' (ie, pleiotropic effects are independent in the magnitude of the SNPexposure associations, and its mean is zero), the effect can be reliably estimated by the multiplicative random effects IVW method. However, if the mean pleiotropic effect is non-zero, as shown by the presence of a deviation from a zero intercept of an MR Egger regression, robust MR methods are indicated. Thus, IVW results were compared with other MR methods to address the violations of specific instrumental variable assumptions: weighted median MR-Pleiotropy RESidual Sum and Outlier (MR-PRESSO) and MR Mixture. The weighted median approach selects the median MR estimate as the causal estimate. ${ }^{29}$ To detect and correct for any outliers reflecting likely pleiotropic biases for all reported results, the MR-PRESSO method was applied. ${ }^{30}$ For sedentary behavior, there were less than five variants in combination with fasting glucose, HOMA-B and HOMA-IR. The MR-PRESSO method is based on the assumption that at least $50 \%$ of the variants are valid instruments relying on the Instrument Strength Independent of Direct Effect (InSIDE) condition. Thus, with less than five SNPs, the MR-PRESSO results were not very meaningful, and therefore, we omitted this analysis. The MRMix approach is a robust MR analysis tool that has the ability to trade off bias and efficiency for estimation of causal effects in the presence of invalid instruments. ${ }^{31}$

We performed leave-one-out analyses and exclusion of potentially pleiotropic SNPs to rule out possible pleiotropic effects (see online supplemental tables 8-10). The study was not preregistered. Analyses were performed using the TwoSampleMR (V.0.4.25) ${ }^{32}$ and MRPRESSO (V.1.0) packages in R (V.3.6.1). Reporting follows the Strengthening the Reporting of Observational Studies in Epidemiology statement. ${ }^{33}$

\section{ETHICS APPROVAL}

In the present study, publicly available summary statistics were used, and therefore, no ethical approval was required.

\section{RESULTS}

\section{Accelerometer-based PA and type 2 diabetes and glycemic} traits

Average accelerations were unrelated to type 2 diabetes (IVW OR per 1 SD: 1.00 ; $95 \%$ CI 0.94 to 1.07 ), HbA1c (IVW $\beta$-estimate per 1 SD: $0.001 ; 95 \%$ CI -0.007 to 0.01 ), fasting glucose (IVW $\beta$-estimate per $1 \mathrm{SD}$ : $-0.001 ; 95 \%$ CI -0.017 to 0.015 ), HOMA-B (IVW $\beta$-estimate per $1 \mathrm{SD}$ : $-0.002 ; 95 \%$ CI -0.021 to 0.017 ), and HOMA-IR (IVW $\beta$-estimate per $1 \mathrm{SD}$ : $-0.001 ; 95 \%$ CI -0.019 to 0.016 (table 1)) across all MR methods.

We also found no evidence that vigorous PA was associated with type 2 diabetes (IVW OR per 1 SD: 0.83 ; $95 \%$ CI 0.56 to 1.23 ). Also, there was no association between vigorous $\mathrm{PA}$ and the investigated glycemic traits $\mathrm{HbAlc}$, fasting glucose, HOMA-B, and HOMA-IR (table 2).

\section{Accelerometer-based SB and type 2 diabetes and glycemic traits}

We found that genetically predicted SB was unrelated to type 2 diabetes (IVW OR per 1 SD: 0.86 ; $95 \%$ CI 0.69 to 1.08 ) and all the other investigated outcomes (table 3 ). This result was confirmed across all MR methods.

We conducted a series of sensitivity analyses to assess the robustness of the results. The $\mathrm{F}$ statistics for the genetic instruments were 30 or higher, consistent with an absence of weak instrument bias (online supplemental table 1). 
Table 1 Mendelian randomization (MR) estimates between accelerometer-based average accelerations and type 2 diabetes and glycemic traits

\begin{tabular}{|c|c|c|c|c|c|}
\hline Method & $\begin{array}{l}\text { No. of } \\
\text { SNPs }\end{array}$ & OR/beta & $95 \% \mathrm{Cl}$ & $P$ value & Outcome \\
\hline $\begin{array}{l}\text { Inverse variance weighted (multiplicative } \\
\text { random effects) }\end{array}$ & 7 & 1.00 & (0.94 to 1.07$)$ & 0.948 & Type 2 diabetes \\
\hline Weighted median & 7 & 1.01 & (0.97 to 1.06$)$ & 0.562 & Type 2 diabetes \\
\hline MR-PRESSO: outlier corrected & 4 & 1.03 & (1.01 to 1.06$)$ & 0.103 & Type 2 diabetes \\
\hline MRMix & 7 & 1.52 & (1.14 to 2.03$)$ & 0.004 & Type 2 diabetes \\
\hline $\begin{array}{l}\text { Inverse variance weighted (multiplicative } \\
\text { random effects) }\end{array}$ & 5 & 0.001 & $(-0.007$ to 0.01$)$ & 0.767 & $\mathrm{HbA1c}$ \\
\hline Weighted median & 5 & 0.000 & ( -0.01 to 0.01$)$ & 0.968 & $\mathrm{HbA1c}$ \\
\hline MR-PRESSO: raw & 5 & 0.001 & $(-0.007$ to 0.01$)$ & 0.782 & $\mathrm{HbA1c}$ \\
\hline MRMix & 5 & 0.035 & $(-0.917$ to 0.987$)$ & 0.943 & $\mathrm{HbA1c}$ \\
\hline $\begin{array}{l}\text { Inverse variance weighted (multiplicative } \\
\text { random effects) }\end{array}$ & 5 & -0.001 & $(-0.017$ to 0.015$)$ & 0.914 & Fasting glucose \\
\hline Weighted median & 5 & 0.002 & $(-0.019$ to 0.023$)$ & 0.861 & Fasting glucose \\
\hline MR-PRESSO: raw & 5 & -0.001 & $(-0.012$ to 0.01$)$ & 0.886 & Fasting glucose \\
\hline MRMix & 5 & -0.010 & $(-0.204$ to 0.184$)$ & 0.919 & Fasting glucose \\
\hline $\begin{array}{l}\text { Inverse variance weighted (multiplicative } \\
\text { random effects) }\end{array}$ & 5 & -0.002 & $(-0.021$ to 0.017$)$ & 0.811 & HOMA-B \\
\hline Weighted median & 5 & -0.010 & (-0.03 to 0.009$)$ & 0.294 & HOMA-B \\
\hline MR-PRESSO: raw & 5 & -0.002 & $(-0.021$ to 0.017$)$ & 0.822 & HOMA-B \\
\hline MRMix & 5 & -0.360 & $(-0.698$ to -0.022$)$ & 0.037 & HOMA-B \\
\hline $\begin{array}{l}\text { Inverse variance weighted (multiplicative } \\
\text { random effects) }\end{array}$ & 5 & -0.001 & $(-0.019$ to 0.016$)$ & 0.871 & HOMA-IR \\
\hline Weighted median & 5 & -0.003 & (-0.026 to 0.02$)$ & 0.786 & HOMA-IR \\
\hline MR-PRESSO: raw & 5 & -0.001 & $(-0.018$ to 0.015$)$ & 0.875 & HOMA-IR \\
\hline MRMix & 5 & -0.010 & $(-0.269$ to 0.249$)$ & 0.940 & HOMA-IR \\
\hline
\end{tabular}

HbA1c, hemoglobin A1c; HOMA-B, homeostasis model assessment of beta-cell function; HOMA-IR, homeostasis model assessment of insulin resistance; MR-PRESSO, MR-Pleiotropy RESidual Sum and Outlier; SNPs, single nucleotide polymorphisms.

The intercept test from MR-Egger regression suggested no presence of pleiotropy (online supplemental table 11). The estimates from the weighted median approach were consistent with the estimates from the IVW models, and the MR-PRESSO test identified no pleiotropic SNPs. Furthermore, the results from the leave-one-out analysis did not reveal any influential SNPs driving the associations (online supplemental table 8-10).

\section{DISCUSSION}

In this MR study, genetic predisposition to accelerometerbased average PA, vigorous PA and SB were not associated with the risk of type 2 diabetes. Furthermore, the present results did not provide clear evidence for a relationship between the different PA measures or SB and insulin resistance, beta-cell function, HbAlc as well as fasting glucose.

Our findings are not consistent with most previous observational studies and meta-analyses showing a protective association of $\mathrm{PA}$ with type 2 diabetes risk. $^{234} \mathrm{~A}$ recent systematic review and dose-response meta-analysis ${ }^{2}$ reported a $39 \%$ risk reduction $(95 \%$ CI 0.51 to 0.74 ) when comparing high with low self-reported vigorous $\mathrm{PA}$, with moderate heterogeneity across the included eight prospective observational studies in that subanalysis $\left(\mathrm{I}^{2}=73 \%\right)$ and varying levels of adjustment for confounding factors. The discrepancies to our results might be attributable to residual confounding due to unmeasured or imprecisely measured confounders in observational studies. Furthermore, our MR analysis reflect long-term/lifelong PA in contrast to studies using short-term self-reported PA habits or PA interventions as exposure. Usually, higher PA is linked to other healthy lifestyle factors (healthier diet, lower prevalence of obesity and smoking). ${ }^{35}$ The ability to disentangle the impact of highly correlated healthy lifestyle habits from each other and from other positive effects associated with PA and subsequently with a lower risk of diabetes (eg, lower blood pressure and weight, improved lipid profile, and mental well-being) may be limited when using conventional multivariable regression methods. ${ }^{36}$ Furthermore, the association between PA and type 2 
Table 2 Mendelian randomization (MR) estimates between accelerometer-based vigorous PA (fraction of accelerations $>425$ milli-gravities) and type 2 diabetes and glycemic traits

\begin{tabular}{|c|c|c|c|c|c|}
\hline Method & $\begin{array}{l}\text { No. of } \\
\text { SNPs }\end{array}$ & OR/beta & $95 \% \mathrm{Cl}$ & $P$ value & Outcome \\
\hline $\begin{array}{l}\text { Inverse variance weighted (multiplicative } \\
\text { random effects) }\end{array}$ & 8 & 0.83 & (0.56 to 1.232$)$ & 0.357 & Type 2 diabetes \\
\hline Weighted median & 8 & 0.80 & (0.58 to 1.11$)$ & 0.177 & Type 2 diabetes \\
\hline MR-PRESSO: outlier corrected & 6 & 0.86 & (0.60 to 1.21$)$ & 0.421 & Type 2 diabetes \\
\hline MRMix & 8 & 1.45 & (0.86 to 2.44 ) & 0.165 & Type 2 diabetes \\
\hline $\begin{array}{l}\text { Inverse variance weighted (multiplicative } \\
\text { random effects) }\end{array}$ & 6 & -0.018 & $(-0.085$ to 0.05$)$ & 0.606 & $\mathrm{HbA1c}$ \\
\hline Weighted median & 6 & 0.016 & (-0.07 to 0.102$)$ & 0.717 & $\mathrm{HbA1c}$ \\
\hline MR-PRESSO: raw & 6 & -0.018 & $(-0.079$ to 0.043$)$ & 0.595 & $\mathrm{HbA1c}$ \\
\hline MRMix & 6 & -0.035 & $(-0.169$ to 0.099$)$ & 0.609 & $\mathrm{HbA1C}$ \\
\hline $\begin{array}{l}\text { Inverse variance weighted (multiplicative } \\
\text { random effects) }\end{array}$ & 6 & 0.065 & $(-0.11$ to 0.24$)$ & 0.469 & Fasting glucose \\
\hline Weighted median & 6 & 0.059 & $(-0.148$ to 0.266$)$ & 0.578 & Fasting glucose \\
\hline MR-PRESSO: raw & 6 & 0.065 & $(-0.11$ to 0.24$)$ & 0.502 & Fasting glucose \\
\hline MRMix & 6 & -0.010 & $(-0.574$ to 0.554$)$ & 0.972 & Fasting glucose \\
\hline $\begin{array}{l}\text { Inverse variance weighted (multiplicative } \\
\text { random effects) }\end{array}$ & 6 & 0.069 & $(-0.071$ to 0.209$)$ & 0.336 & HOMA-B \\
\hline Weighted median & 6 & 0.013 & $(-0.17$ to 0.197$)$ & 0.886 & HOMA-B \\
\hline MR-PRESSO: raw & 6 & 0.069 & (-0.071 to 0.209$)$ & 0.380 & HOMA-B \\
\hline MRMix & 6 & 0.110 & $(-0.902$ to 1.122$)$ & 0.831 & HOMA-B \\
\hline $\begin{array}{l}\text { Inverse variance weighted (multiplicative } \\
\text { random effects) }\end{array}$ & 6 & 0.102 & $(-0.111$ to 0.316$)$ & 0.349 & HOMA-IR \\
\hline Weighted median & 6 & -0.041 & $(-0.258$ to 0.176$)$ & 0.708 & HOMA-IR \\
\hline MR-PRESSO: raw & 6 & 0.102 & $(-0.111$ to 0.316$)$ & 0.392 & HOMA-IR \\
\hline MRMix & 6 & -0.030 & $(-0.455$ to 0.395$)$ & 0.890 & HOMA-IR \\
\hline
\end{tabular}

HbA1c, hemoglobin A1c; HOMA-B, homeostasis model assessment of beta-cell function; HOMA-IR, homeostasis model assessment of insulin resistance ; MR-PRESSO, MR-Pleiotropy RESidual Sum and Outlier; PA, physical activity; SNPs, single nucleotide polymorphisms.

diabetes in observational studies might be due to reverse causation. Individuals screened for high risk of type 2 diabetes or with a diagnosis of pre-diabetes possibly change their lifestyle to delay or prevent a manifestation of the disease. ${ }^{37} 38 \mathrm{~A}$ change in lifestyle might include increasing levels of PA in addition to diet changes, weight loss, and quitting smoking. ${ }^{37} 38$

In an exposure-wide umbrella review of meta-analyses (including observational studies) regarding risk factors for type 2 diabetes, the association between sedentary time and risk of type 2 diabetes was supported by convincing evidence. ${ }^{39}$ Another systematic review and meta-analysis on the association between self-reported SB and different health outcomes found an increased risk for incident type 2 diabetes with higher levels of total sitting as well as TV viewing time, independent of PA. ${ }^{40}$ This finding was not replicated in the present study. However, in that meta-analysis, there was substantial heterogeneity in exposure measurement and unmeasured confounding was likely, ${ }^{40}$ and thus it is difficult to ascertain causality. It is conceivable that in SB studies, confounding by socioeconomic status is more likely than in PA studies because TV time is strongly negatively associated with education level. ${ }^{41}$

A number of randomized controlled trials (RCTs) have examined the effect of exercise interventions on glycemic traits. However, the majority of these RCTs used an intervention that combined exercise and diet components, which make it difficult to attribute the effect to $\mathrm{PA}^{42}$; only one RCT focused exclusively on PA intervention. ${ }^{43}$ Recently, a Cochrane Intervention Review of 12 RCTs with a total of 5238 persons on the issue whether $\mathrm{PA}$, diet or both can prevent or delay type 2 diabetes and its associated complications was published. ${ }^{42}$ Most of the trials included persons at increased risk of type 2 diabetes and the duration of the intervention varied between 2 and 6 years. Only one trial compared diet with PA in one of its trial arms. ${ }^{44}$ There was no clear evidence that diet alone or PA alone compared with standard treatment influences the risk for type 2 diabetes, ${ }^{42}$ and the overall quality of evidence was very low. Our study confirms this finding by showing that objectively measured PA of 
Table 3 Mendelian randomization (MR) estimates between accelerometer-based sedentary behavior (MET $\leq 1.5)$ and type 2 diabetes and glycemic traits

\begin{tabular}{|c|c|c|c|c|c|}
\hline Method & $\begin{array}{l}\text { No. of } \\
\text { SNPs }\end{array}$ & OR/beta & $95 \% \mathrm{Cl}$ & $P$ value & Outcome \\
\hline $\begin{array}{l}\text { Inverse variance weighted (multiplicative } \\
\text { random effects) }\end{array}$ & 5 & 0.86 & (0.69 to 1.08$)$ & 0.190 & Type 2 diabetes \\
\hline Weighted median & 5 & 0.80 & (0.60 to 1.08$)$ & 0.141 & Type 2 diabetes \\
\hline MR-PRESSO: raw & 6 & 0.86 & (0.69 to 1.08$)$ & 0.260 & Type 2 diabetes \\
\hline MRMix & 6 & 1.00 & (0.58 to 1.73$)$ & 1.000 & Type 2 diabetes \\
\hline $\begin{array}{l}\text { Inverse variance weighted (multiplicative } \\
\text { random effects) }\end{array}$ & 4 & 0.010 & $(-0.078$ to 0.099$)$ & 0.818 & $\mathrm{HbA1c}$ \\
\hline Weighted median & 4 & 0.030 & $(-0.069$ to 0.129$)$ & 0.553 & $\mathrm{HbA1c}$ \\
\hline MR-PRESSO: raw & 5 & 0.010 & $(-0.078$ to 0.099$)$ & 0.833 & $\mathrm{HbA1c}$ \\
\hline MRMix & 5 & 0.025 & $(-0.130$ to 0.180$)$ & 0.751 & $\mathrm{HbA} 1 \mathrm{c}$ \\
\hline $\begin{array}{l}\text { Inverse variance weighted (multiplicative } \\
\text { random effects) }\end{array}$ & 3 & 0.014 & $(-0.142$ to 0.171$)$ & 0.856 & Fasting glucose \\
\hline Weighted median & 3 & 0.045 & $(-0.144$ to 0.234$)$ & 0.643 & Fasting glucose \\
\hline MRMix & 4 & 0.025 & $(-0.185$ to 0.235$)$ & 0.816 & Fasting glucose \\
\hline $\begin{array}{l}\text { Inverse variance weighted (multiplicative } \\
\text { random effects) }\end{array}$ & 3 & 0.001 & $(-0.264$ to 0.267$)$ & 0.991 & HOMA-B \\
\hline Weighted median & 3 & 0.126 & $(-0.068$ to 0.319$)$ & 0.203 & HOMA-B \\
\hline MRMix & 4 & 0.230 & $(-0.122$ to 0.582$)$ & 0.200 & HOMA-B \\
\hline $\begin{array}{l}\text { Inverse variance weighted (multiplicative } \\
\text { random effects) }\end{array}$ & 3 & 0.023 & $(-0.363$ to 0.409$)$ & 0.907 & HOMA-IR \\
\hline Weighted median & 3 & 0.113 & $(-0.154$ to 0.38$)$ & 0.407 & HOMA-IR \\
\hline MRMix & 4 & 0.320 & $(-0.078$ to 0.718$)$ & 0.115 & HOMA-IR \\
\hline
\end{tabular}

HbA1c, hemoglobin A1c; HOMA-B, homeostasis model assessment of beta-cell function; HOMA-IR, homeostasis model assessment of insulin resistance; MET, metabolic equivalent task; MR-PRESSO, MR-Pleiotropy RESidual Sum and Outlier; SNPs, single nucleotide polymorphisms.

different intensities alone does not significantly reduce the risk of type 2 diabetes.

We also found no associations between objectively measured PA levels or SB and various glycemic traits. RCTs on this topic have yielded inconsistent results. In a systematic review and meta-analysis of randomized trials including 7487 participants aged 18-90 years (78.1\% free of diabetes) based on 160 RCTs, moderate to long duration exercise training (2 weeks-2 years ${ }^{45}$ had a protective effect on insulin resistance (HOMA-IR) and HbA1c but not on fasting glucose levels. ${ }^{45}$ Other systematic reviews and meta-analyses also suggested a protective effect of PA on insulin resistance. However, the quality of the included studies in those meta-analyses was mostly poor (high risk of bias) ${ }^{46}$ and the quality of evidence was low or very low for all outcomes. ${ }^{47}$

In addition, a systematic review and meta-analysis reported inverse relations of $\mathrm{HbAlc}$ and insulin to resistance exercise training intensity, ${ }^{47}$ and other metaanalyses found a reduction in fasting glucose levels after resistance exercise interventions in non-diabetic persons ${ }^{47}$ or a reduction of fasting glucose and HbAlc in physically active persons with type 2 diabetes or metabolic syndrome only. ${ }^{46}$ Another systematic review and meta-analysis supported a decrease in HbA1c levels in favor of the physically active group (effect size 0.32; $95 \%$ CI 0.01 to 0.62 ) noting substantial heterogeneity $\left(\mathrm{I}^{2}=63.2 \% ; \mathrm{p}=0.008\right) .{ }^{48}$

Our MR analysis found no effect of average PA, vigorous $\mathrm{PA}$ and $\mathrm{SB}$ on glycemic traits. This seems plausible because it is unlikely that PA will have a significant effect on these glycemic traits in the normal range in non-diabetic individuals. Most previous RCTs investigating the effects of PA on fasting glucose and HbAlc levels were conducted in high-risk groups, such as individuals with pre-diabetes or obesity or people with manifest type 2 diabetes. Hence, from observational studies and RCTs on this issue, it is not clear so far whether associations between PA and type 2 diabetes are causal or biased due to self-report measurement error, residual confounding, reverse causality, or ascertainment bias in RCTs.

The findings of our two-sample MR study regarding the outcome type 2 diabetes are in line with results reported by Doherty et al. ${ }^{15}$ In that study, the MR analysis on the causal association between moderate accelerometer measured PA and diabetes in UK Biobank participants who were not in the accelerometer discovery dataset 
resulted in an OR for diabetes of $0.86(p=0.079)$, indicating no causal effect.

\section{Strengths and limitations}

Contrary to observational studies and RCTs for PA, the major advantage of our study is the use of MR, which has the ability to reflect lifelong exposure to a causal factor that might differ from self-reported PA habits or PA interventions for shorter time periods later in life. A further advantage of our study is the two-sample MR study design, diminishing unobserved confounding and reverse causality. The very large sample size (almost 900 000 individuals in the type 2 diabetes outcome study, almost 500000 individuals for PA) provided the power to detect effect sizes previously reported in observational studies and to perform multiple sensitivity analyses for testing the validity of the MR assumptions, thus minimizing the possibility of biased results. Our study also has limitations. Because only data from European populations was used, our findings may differ in other ethnicities. The outcome type 2 diabetes may be affected by a degree of misclassification because case ascertainment in the studies included in the DIAGRAM Consortium was not carried out according to certain specifications and thus a broad spectrum of type 2 diabetes cases (those with or without complications, different disease durations and so on) was included. The appeal of accelerometers to objectively monitor PA is their ability to quantify ambulatory activity during walking, jogging and so on. However, accelerometers are not without limitations. The disadvantages of accelerometers are their difficulties to measure posture and sedentary, light activites and non-ambulatory activites (cycling and weightlifting), and for estimating energy expenditure.$^{49}$ Furthermore, awareness that PA is being monitored might influence habitual behavior. ${ }^{50}$

\section{CONCLUSIONS}

In summary, the present two-sample MR study found no evidence of a causal association between genetically determined objectively measured PA and SB with the risk of type 2 diabetes and glycemic traits. There is no question that PA has a positive impact on health, but it seems that the complex interplay of the numerous metabolic effects and multiple biological mechanisms mediating the beneficial role of PA on disease development ${ }^{51}$ are not fully understood so far. Further studies are necessary to deepen our understanding of the biological pathways of PA.

\author{
Author affiliations \\ ${ }^{1}$ Chair of Epidemiology at UNIKA-T Augsburg, Ludwig-Maximilians-Universitat \\ Munchen, Munchen, Germany \\ ${ }^{2}$ Independent Research Group Clinical Epidemiology, Helmholtz Zentrum München, \\ German Research Center for Environmental Health, Neuherberg, Germany \\ ${ }^{3}$ Department of Epidemiology and Preventive Medicine, University of Regensburg, \\ Regensburg, Germany
}

Contributors $\mathrm{CM}, \mathrm{SEB}$, and $\mathrm{HB}$ designed the work and interpreted the data; $\mathrm{HB}$ conducted the analysis; CM drafted the work; all authors critically revised the work and approved the submitted version. All authors have agreed both to be personally accountable for the author's own contributions and to ensure that questions related to the accuracy or integrity of any part of the work, even ones in which the author was not personally involved, are appropriately investigated, resolved, and the resolution documented in the literature.

Funding The authors have not declared a specific grant for this research from any funding agency in the public, commercial or not-for-profit sectors.

Competing interests None declared.

\section{Patient consent for publication Not required.}

Ethics approval The UK Biobank study was ethically approved by the North West Multicentre Research Ethics Committee, the National Information Governance Board for Health \& Social Care, and the Community Health Index Advisory Group.

Provenance and peer review Not commissioned; externally peer reviewed.

Data availability statement Data are available in a public, open access repository. The present study is based on summary-level data that have been made publically available. In all original studies, ethical approval had been obtained. The summary statistics for the PA and SB GWAS is available at https://klimentidis. lab.arizona.edu/content/data and at https://doi.org/10.5287/bodleian:yJp6zZmdj. The summary data for the type 2 diabetes GWAS is available at http://diagramconsortium.org/downloads.html, for the HbA1c, fasting glucose, HOMA-B and HOMA-IR GWASs at www. magicinvestigators.org/downloads.

Supplemental material This content has been supplied by the author(s). It has not been vetted by BMJ Publishing Group Limited (BMJ) and may not have been peer-reviewed. Any opinions or recommendations discussed are solely those of the author(s) and are not endorsed by BMJ. BMJ disclaims all liability and responsibility arising from any reliance placed on the content. Where the content includes any translated material, BMJ does not warrant the accuracy and reliability of the translations (including but not limited to local regulations, clinical guidelines, terminology, drug names and drug dosages), and is not responsible for any error and/or omissions arising from translation and adaptation or otherwise.

Open access This is an open access article distributed in accordance with the Creative Commons Attribution Non Commercial (CC BY-NC 4.0) license, which permits others to distribute, remix, adapt, build upon this work non-commercially, and license their derivative works on different terms, provided the original work is properly cited, appropriate credit is given, any changes made indicated, and the use is non-commercial. See: http://creativecommons.org/licenses/by-nc/4.0/.

\section{ORCID iDs}

Christa Meisinger http://orcid.org/0000-0002-9026-6544

Jakob Linseisen http://orcid.org/0000-0002-9386-382X

\section{REFERENCES}

1 Saeedi P, Petersohn I, Salpea P, et al. Global and regional diabetes prevalence estimates for 2019 and projections for 2030 and 2045: results from the International diabetes Federation diabetes atlas, 9th edition. Diabetes Res Clin Pract 2019;157:107843.

2 Aune D, Norat T, Leitzmann M, et al. Physical activity and the risk of type 2 diabetes: a systematic review and dose-response metaanalysis. Eur J Epidemiol 2015;30:529-42.

3 Boniol M, Dragomir M, Autier P, et al. Physical activity and change in fasting glucose and $\mathrm{HbA1c}$ : a quantitative meta-analysis of randomized trials. Acta Diabetol 2017;54:983-91.

4 Prince SA, Adamo KB, Hamel M, et al. A comparison of direct versus self-report measures for assessing physical activity in adults: a systematic review. Int J Behav Nutr Phys Act 2008;5:56.

5 Folley S, Zhou A, Hyppönen E. Information bias in measures of selfreported physical activity. Int J Obes 2018;42:2062-3.

6 Dyrstad SM, Hansen BH, Holme IM, et al. Comparison of selfreported versus accelerometer-measured physical activity. Med Sci Sports Exerc 2014;46:99-106.

7 Vancampfort D, De Hert M, Myin-Germeys I, et al. Validity and correlates of the International physical activity questionnaire in firstepisode psychosis. Early Interv Psychiatry 2019;13:562-7.

8 Prince SA, Cardilli L, Reed JL, et al. A comparison of self-reported and device measured sedentary behaviour in adults: a systematic review and meta-analysis. Int J Behav Nutr Phys Act 2020;17:31.

9 Doherty A, Jackson D, Hammerla N, et al. Large scale population assessment of physical activity using wrist worn accelerometers: the UK Biobank study. PLoS One 2017;12:e0169649.

10 Smith GD, Ebrahim S. 'Mendelian randomization': can genetic epidemiology contribute to understanding environmental determinants of disease? Int J Epidemiol 2003;32:1-22. 
11 Lawlor DA, Wade K, Borges MC, et al. A Mendelian randomization dictionary: useful definitions and descriptions for undertaking, understanging and interpreting Mendelian randomization studies. Available: https://osf.io/6yzs7/ [Accessed 13 Jun 2020].

12 Klimentidis YC, Raichlen DA, Bea J, et al. Genome-Wide association study of habitual physical activity in over 377,000 UK Biobank participants identifies multiple variants including CADM2 and APOE. Int $J$ Obes 2018;42:1161-76.

13 Davies NM, Holmes MV, Davey Smith G. Reading Mendelian randomisation studies: a guide, glossary, and checklist for clinicians. BMJ 2018;362:k601.

14 Fry A, Littlejohns TJ, Sudlow C, et al. Comparison of sociodemographic and health-related characteristics of UK Biobank participants with those of the general population. Am J Epidemiol 2017; 186:1026-34.

15 Doherty A, Smith-Byrne K, Ferreira T, et al. Gwas identifies 14 loci for device-measured physical activity and sleep duration. Nat Commun 2018;9:5257.

16 Bycroft C, Freeman C, Petkova D, et al. The UK Biobank resource with deep phenotyping and genomic data. Nature 2018;562:203-9.

17 Kamat MA, Blackshaw JA, Young R, et al. PhenoScanner V2: an expanded tool for searching human genotype-phenotype associations. Bioinformatics 2019;35:4851-3.

18 Kaczynski AT, Manske SR, Mannell RC, et al. Smoking and physical activity: a systematic review. Am J Health Behav 2008;32:93-110.

19 Willi C, Bodenmann P, Ghali WA, et al. Active smoking and the risk of type 2 diabetes: a systematic review and meta-analysis. JAMA 2007;298:2654-64.

20 Mahajan A, Taliun D, Thurner M, et al. Fine-Mapping type 2 diabetes loci to single-variant resolution using high-density imputation and islet-specific epigenome maps. Nat Genet 2018;50:1505-13.

21 Wheeler E, Leong A, Liu C-T, et al. Impact of common genetic determinants of hemoglobin A1c on type 2 diabetes risk and diagnosis in ancestrally diverse populations: a transethnic genomewide meta-analysis. PLoS Med 2017;14:e1002383.

22 Manning AK, Hivert M-F, Scott RA, et al. A genome-wide approach accounting for body mass index identifies genetic variants influencing fasting glycemic traits and insulin resistance. Nat Genet 2012;44:659-69.

23 Dupuis J, Langenberg C, Prokopenko I, et al. New genetic loci implicated in fasting glucose homeostasis and their impact on type 2 diabetes risk. Nat Genet 2010;42:105-16.

24 Burgess S. Sample size and power calculations in Mendelian randomization with a single instrumental variable and a binary outcome. Int J Epidemiol 2014;43:922-9.

25 Deng L, Zhang H, Yu K. Power calculation for the general twosample Mendelian randomization analysis. Genet Epidemiol 2020;44:290-9.

26 Burgess S, Davey Smith G, Davies NM, et al. Guidelines for performing Mendelian randomization investigations. Wellcome Open Res 2019;4:186

27 Willetts M, Hollowell S, Aslett L, et al. Statistical machine learning of sleep and physical activity phenotypes from sensor data in 96,220 UK Biobank participants. Sci Rep 2018;8:7961.

28 Ainsworth BE, Haskell WL, Herrmann SD, et al. 2011 compendium of physical activities: a second update of codes and Met values. Med Sci Sports Exerc 2011;43:1575-81.

29 Bowden J, Davey Smith G, Haycock PC, et al. Consistent estimation in Mendelian randomization with some invalid instruments using a weighted median estimator. Genet Epidemiol 2016;40:304-14.

30 Verbanck M, Chen C-Y, Neale B, et al. Detection of widespread horizontal pleiotropy in causal relationships inferred from Mendelian randomization between complex traits and diseases. Nat Genet 2018;50:693-8.

31 Qi G, Chatterjee N. Mendelian randomization analysis using mixture models for robust and efficient estimation of causal effects. Nat Commun 2019;10:1941.
32 Hemani G, Zheng J, Elsworth B, et al. The MR-Base platform supports systematic causal inference across the human phenome. Elife 2018;7:e34408

33 Smitz GD, Davies NM, Dimou N, et al. STROBE-MR: guidelines for strengthening the reporting of Mendelian randomization studies. PeerJ Preprints 2019;7:e27857v1 https://doi.org/10.7287/peerj. preprints.27857v1

34 Smith AD, Crippa A, Woodcock J, et al. Physical activity and incident type 2 diabetes mellitus: a systematic review and doseresponse meta-analysis of prospective cohort studies. Diabetologia 2016;59:2527-45.

35 Aune D, Ursin G, Veierød MB. Meat consumption and the risk of type 2 diabetes: a systematic review and meta-analysis of cohort studies. Diabetologia 2009;52:2277-87.

36 Phillips AN, Smith GD. How independent are "independent" effects? relative risk estimation when correlated exposures are measured imprecisely. J Clin Epidemiol 1991;44:1223-31.

37 Elman K, Wainstein J, Boaz M, et al. Random blood glucose screening at a public health station encouraged high risk subjects to make lifestyle changes. Int J Clin Pract 2017;71:e12984.

38 Owei I, Umekwe N, Ceesay F, et al. Awareness of prediabetes status and subsequent health behavior, body weight, and blood glucose levels. J Am Board Fam Med 2019;32:20-7.

39 Bellou V, Belbasis L, Tzoulaki I, et al. Risk factors for type 2 diabetes mellitus: an exposure-wide umbrella review of meta-analyses. PLoS One 2018;13:e0194127.

40 Patterson R, McNamara E, Tainio M, et al. Sedentary behaviour and risk of all-cause, cardiovascular and cancer mortality, and incident type 2 diabetes: a systematic review and dose response metaanalysis. Eur J Epidemiol 2018;33:811-29.

41 van de Vegte YJ, Said MA, Rienstra M, et al. Genome-Wide association studies and Mendelian randomization analyses for leisure sedentary behaviours. Nat Commun 2020;11:1770.

42 Hemmingsen B, Gimenez-Perez G, Mauricio D, et al. Diet, physical activity or both for prevention or delay of type 2 diabetes mellitus and its associated complications in people at increased risk of developing type 2 diabetes mellitus. Cochrane Database Syst Rev 2017;12:CD003054.

43 Hellgren MI, Jansson P-A, Wedel H, et al. A lifestyle intervention in primary care prevents deterioration of insulin resistance in patients with impaired glucose tolerance: a randomised controlled trial. Scand J Public Health 2016:44:718-25.

$44 \mathrm{Li} \mathrm{G}$, Zhang P, Wang J, et al. The long-term effect of lifestyle interventions to prevent diabetes in the China dA Qing diabetes prevention study: a 20-year follow-up study. The Lancet 2008;371:1783-9.

45 Lin X, Zhang X, Guo J, et al. Effects of exercise training on cardiorespiratory fitness and biomarkers of cardiometabolic health: a systematic review and meta-analysis of randomized controlled trials. $J$ Am Heart Assoc 2015:4:e002014.

46 Jelleyman C, Yates T, O'Donovan G, et al. The effects of highintensity interval training on glucose regulation and insulin resistance: a meta-analysis. Obes Rev 2015;16:942-61.

47 Ashton RE, Tew GA, Aning JJ, et al. Effects of short-term, mediumterm and long-term resistance exercise training on cardiometabolic health outcomes in adults: systematic review with meta-analysis. $\mathrm{Br}$ $J$ Sports Med 2020;54:341-8.

48 Cavero-Redondo I, Peleteiro B, Álvarez-Bueno C, et al. The Effect of Physical Activity Interventions on Glycosylated Haemoglobin $\left(\mathrm{HbA}_{11}\right)$ in Non-diabetic Populations: A Systematic Review and Metaanalysis. Sports Med 2018;48:1151-64.

49 Lee I-M, Shiroma EJ. Using accelerometers to measure physical activity in large-scale epidemiological studies: issues and challenges. Br J Sports Med 2014;48:197-201.

50 Pedišić Željko, Bauman A. Accelerometer-based measures in physical activity surveillance: current practices and issues. $\mathrm{Br} \mathrm{J}$ Sports Med 2015;49:219-23.

51 Thyfault JP, Bergouignan A. Exercise and metabolic health: beyond skeletal muscle. Diabetologia 2020;63:1464-74. 